\title{
De víctimas a activistas expertos: Marco conceptual para el estudio del devenir militante en la configuración del campo de derechos humanos en el Chile actual
}

\author{
Manuel Guerrero \\ Universidad de Chile \\ mguerrero@med.uchile.cl
}

RESUMEN: Si bien es significativo el valor que las ciencias sociales y las humanidades le han asignado al movimiento de derechos humanos en América Latina, como uno de los elementos explicativos para las transiciones de gobiernos autoritarios a las nuevas democracias y a la consolidación de las mismas, en general no se ha puesto suficiente atención a las condiciones sociales de producción y reproducción del activismo en derechos humanos en el tiempo, que no sea un supuesto vago de que habría en juego relaciones de parentesco con víctimas del terrorismo de Estado como factor de adhesión y movilización de sus miembros. De este modo, el compromiso militante con la causa y la acción colectiva en este campo aparecen como dados, quedando sin investigar el proceso de acercamiento, ingreso, permanencia, desarrollo, salida y eventual reincorporación -el "devenir militante" en el movimiento de derechos humanos-. Este artículo busca aportar algunas claves de acceso a este campo de investigación que permitan superar la matriz de comprensión del "modelo familiar".

Palabras Clave: movimientos sociales, devenir militante, derechos humanos, memoria social. 
FrOM ACTIVISTS TO EXPERTS: KEYS FOR THE STUDY OF BECOMING MILITANT IN THE CONFIGURATION OF THE HUMAN RIGHTS' FIELD IN CONTEMPORARY CHILE

Abstract: Although the value assigned by the social sciences and the humanities to the human rights movement in Latin America as one of the explanatory elements for the transition from authoritarian governments to new democracies -and their consolidation- is noteworthy, not enough attention has been paid to the social conditions of production and reproduction over time of human rights activism, apart from a vague presupposition that a kinship relationship with victims of state terrorism would be at stake in the support and mobilisation of its members. Thus, militant commitment to the cause and collective action in this field appear as given, with the process of approach, entry, permanence, development, exit and eventual reincorporation -the "becoming militant" in the human rights movement- remaining uninvestigated. This paper aims to contribute to some key lines of access to this research field that allow us to overcome the "family model" matrix of understanding.

KEYwords: Social movements, becoming militant, human rights, social memory.

LUCES Y SOMBRAS EN LA COMPRENSIÓN DEL MOVIMIENTO DE DERECHOS HUMANOS COMO FORMA DE ACCIÓN COLECTIVA

Las perspectivas de análisis de la acción colectiva desde las ciencias sociales se han vinculado, en forma preferente, a la comprensión de los movimientos sociales en una clave sustancialista, que considera al colectivo como una unidad homogénea. Por una parte, la acción colectiva tendió a ser estudiada con énfasis en la racionalidad del actor -esto es, desde el cálculo (presupuesto) que realizan los individuos organizados en colectivo-, respecto de las ventajas y costos de la acción colectiva, movidos por incentivos positivos o negativos (Olson). Por otra parte, se ha apuntado al determinismo estructural, es decir, se ha recurrido a factores externos (contexto político, nivel de represión del Estado, estructuras sociales) como variables de análisis que explican las condiciones de posibilidad de un movimiento social (Smelser).

En los años sesenta se puso énfasis en teorías ligadas al ámbito de la psicología social, que explicaban el compromiso militante desde la teoría 
de la frustración relativa, levantando el modelo del militante irracional y anómico (Davies), cuyos orígenes se encuentran ya en las tesis de Gustave Le Bon y Gabriel Tarde sobre la "irracionalidad de las masas". Posteriormente, en los años setenta, con la emergencia de la teoría de la movilización de recursos, la explicación se sofisticó un poco más, desplazándose paulatinamente a modelos que enfatizaban las "estructuras de oportunidad política" que relevaban factores adicionales de contexto determinantes para la acción colectiva (Tarrow; Kitschelt 57; McAdam; Kriesi; McAdam, McCarthy y Zald; McAdam, Tarrow y Tilly).

En otra línea de análisis se desarrolla la teoría de Snow sobre los marcos cognitivos (Snow y Benford, "Master frames"; Snow et al., "Frame alignment" 51), desde la cual se postula la capacidad de los movimientos sociales para crear "cosmovisiones" compartidas entre sus miembros, que operan como marcos de referencia a través de los cuales se perciben las oportunidades. Así es como se producen metáforas específicas, representaciones simbólicas e indicaciones cognitivas que se utilizan para representar conductas y eventos en forma evaluativa y para sugerir formas alternativas de acción. Los "marcos cognitivos" se definen como los discursos culturales que posibilitan la descripción de significados compartidos que impulsan a las personas a la acción colectiva (Touraine).

No obstante estas variaciones, por lo general, al no existir análisis a escala de la experiencia de los individuos y sus interacciones, el estudio de las formas o condiciones de posibilidad de las organizaciones políticas y de las movilizaciones colectivas se ha limitado a niveles de análisis macro y meso sociológicos. Lo anterior ha impedido comprender el peso sociológico del itinerario que lleva a alguien a comprometerse para actuar, mantener el compromiso adquirido, eventualmente abandonarlo e incluso reingresar (Fillieule, "Propositions" 199; Bennani-Chraibi y Fillieule).

Esto es lo que ha sucedido también en el caso del estudio del movimiento de derechos humanos. En efecto, la explicación otorgada generalmente desde la ciencia política y la sociología de los movimientos sociales es que el movimiento de derechos humanos en Chile y Latinoamérica se moviliza, fundamentalmente, a partir de su constitución por lazos de parentesco con las víctimas de la represión del terrorismo de Estado (Fuentes; Valenzuela 127), con la voluntad de acelerar la vuelta a la democracia en regímenes autoritarios o exigir sanción a los culpables de los atropellos y reparación a las víctimas en las nuevas democracias (Hipsher 153; 
O'Donnell y Schmitter; Oxhorn; Schneider). A este respecto, es claro que hace falta interrogarse en forma desprejuiciada acerca de las condiciones sociales de producción y reproducción de este activismo, sobre quiénes lo integran, sus experiencias e interacciones y sobre sus diversas lógicas de funcionamiento en el tiempo (Vecchioli "Os Trábalhos"; Vecchioli "Derechos Humanos"; Groppo).

En Chile, los estudios sobre el campo de los derechos humanos -en lo que respecta a los movimientos, agrupaciones y asociaciones- se remontan a los trabajos desarrollados en el período de la dictadura y a los diez primeros años de la nueva democracia. La mayoría trata sobre el registro de la represión y el testimonio en torno a ella, el papel de las organizaciones como un todo, así como balances de las políticas públicas de la nueva democracia (Vidal, El Movimiento; Edina; Frühling; Orellana; Palestro; Brett; Vidal, Dar la vida; AFDD; Brito; Loveman; Lira 339; Hawkins). No existe material que investigue la composición interna del campo y de las organizaciones de defensa de los derechos humanos, en particular en lo que respecta a los agentes sociales que las componen y las relaciones entre ellos de acuerdo a sus propiedades sociales y trayectorias militantes. Generalmente, los textos abordan la descripción de los principales organismos de derechos humanos surgidos durante la dictadura en reacción a la represión ejercida por el terrorismo de Estado (Vicaría de la Solidaridad, Fundación de Ayuda Social de las Iglesias Cristianas, Servicio Paz y Justicia, Programa de Derechos Humanos de la Academia de Humanismo Cristiano, Comisión Chilena de Derechos Humanos, Comisión Nacional Pro Derechos Juveniles, Comité de Defensa de los Derechos del Pueblo, Comisión Nacional Contra la Tortura), y en lo que toca a los movimientos, agrupaciones y asociaciones se centran preferentemente en las organizaciones de familiares (Agrupación de Familiares de Detenidos Desaparecidos, Agrupación de Familiares de Presos Políticos, Comité Pro-retorno de Exiliados, Agrupación de Familiares de Ejecutados Políticos), con la excepción del Movimiento Contra la Tortura Sebastián Acevedo ${ }^{1}$.

Un trabajo muy completo que, no quedándose en una descripción general, avanza en una caracterización más compleja de los organismos de derechos humanos, es el de Orellana y Hutchison, que contempla la dependencia, orientación, afiliaciones, apoyo internacional, división del trabajo, cobertura regional, política de publicaciones 
LA SOCIOLOGÍA DE LA MILITANCIA COMO HERRAMIENTA HEURÍSTICA PARA EL ESTUDIO DEL CAMPO DE DERECHOS HUMANOS

Como no existen investigaciones previas en Chile que consideren las biografías sociales y políticas de los agentes sociales en general y de los grupos dirigentes en particular, y su movimiento al interior del campo de los derechos humanos, postulo que este emergente campo de investigación puede apoyarse, como herramienta heurística, en el acervo de investigaciones que se han comenzado a realizar sobre el compromiso militante y la acción colectiva a partir de los años noventa en Europa, y de modo reciente en América Latina: la "sociología de la militancia". Estas investigaciones han permitido avanzar hacia la renovación del estudio de las organizaciones políticas y de las movilizaciones en relación con nuevos contextos históricos y sociales marcados por el declive de la adhesión a los partidos políticos. En una perspectiva crítica de los teóricos del proceso político, y sobre la base de una gran diversidad de trabajos de campo cualitativos y cuantitativos, la utilización de estas nuevas perspectivas ha permitido demostrar que ni las teorías del comportamiento colectivo -que se basan en la predeterminación de la acción-, ni la escuela de la movilización de recursos -que opera desde la metáfora de la racionalidad del actor-, así como tampoco las aproximaciones estructuralistas del proceso político han desarrollado, por sí mismas, un modelo explicativo convincente acerca del rol de los individuos en el desarrollo de la acción colectiva, aspecto que resulta fundamental tener en cuenta para no naturalizar el papel de las estructuras, ni tampoco huir al otro extremo: el de la subjetividad pura.

La experiencia de los individuos y sus interacciones al interior de un espacio social que establece "lo(s) posible(s)" -aquella zona oscura de la acción colectiva- aparece cada vez con mayor fuerza como un campo de estudio clave para poder comprender la trayectoria de las organizaciones

y comunicaciones. Lamentablemente, dado el momento en que fue realizado el libro y los marcos teóricos a la mano, los autores solo les dedican una página a los procesos de vinculación de quienes ingresaban a estos organismos, quedando sin investigar los procesos sociales que están en la base para que distintas personas decidan ingresar a aquellos, desarrollen un compromiso militante con la causa y la acción colectiva, y avancen en carreras al interior de sus organizaciones. Por otra parte, el texto aborda solo el periodo 1973-1990. 
políticas, su génesis, permanencia, reconversión o declive. A este respecto, las nuevas investigaciones orientadas a examinar y comprender las trayectorias militantes permiten dar cuenta del compromiso individual y sus transformaciones en el curso de la acción, lo cual puede ser analizado como un fenómeno variable en intensidad y duración en función de, al menos, tres tipos de variables:

- Variables contextuales estructurales, como el sistema de disposiciones duraderas, determinadas por el capital social, cultural y económico del individuo que contribuye a modelar las percepciones de la realidad y las prácticas (Bourdieu y Wacquant), así como el contexto político (arreglos institucionales, niveles de represión, etc.);

- Variables contextuales relacionales, como las redes de relaciones en las que el individuo está inserto, que tienen un efecto doble sobre los procesos de movilización -en tanto instancias de socialización y de conversión-, que ponen a disposición cuadros interpretativos del mundo y producen oportunidades de movilización;

- Y la trayectoria del individuo que se despliega en el marco del contexto estructural y de redes sociales preexistentes, que involucra tomas de posición y puesta en juego de estrategias.

Con este enfoque se ha abierto una nueva agenda de investigación, que no se centra solo en factores estructurales, contextos políticos y dinámicas organizacionales, sino también en el individuo que participa en la acción colectiva: el militante, el adherente y las redes en las cuales se encuentran insertos ${ }^{2}$.

2 Algunos sociólogos y cientistas políticos que investigan, que han publicado y trabajan en esta línea desde mitad de los años noventa son Olivier Fillieule (Lausanne/ CRPS-Universidad Paris I), Eric Agrikoliansky (CREDEP-Universidad Paris IX), Nonna Mayer (GERMM-Groupe d'étude et de recherche sur les mutations du militantisme), Bernard Pudal (Université Paris-X Nanterre). En Chile, Stéphanie Alenda ha investigado, en parte basándose en este enfoque y aplicándolo desde las ciencias políticas, sobre la UDI, RN y el PDC (INAP-Universidad de Chile) y Daniela Cuadros Garland (Université de Paris I) sobre las reconversiones de los abogados de derechos humanos. Al respecto ver: Cuadros (37-51). 
Desde este enfoque se asume la idea de la militancia como una actividad individual y colectiva inscrita en el tiempo y en el espacio, que articula fases de adhesión, mantención del compromiso y abandono, perspectiva para la cual el concepto de "carrera", proveniente de la tradición interaccionista de la Escuela de Chicago, resulta una herramienta heurística valiosa ${ }^{3}$.

En efecto, la noción de carrera permite pensar relacionalmente variables relativas a la predisposición a la militancia, la entrada a la misma, las formas diferenciales y variables del compromiso en el tiempo, la multiplicidad de compromisos a lo largo de un ciclo de vida -como ingreso, retiro(s) y desplazamiento(s) de un colectivo a otro, de un tipo de militancia a otra, de un campo a otro-, y la retracción o extensión del compromiso militante adquirido.

Con ello, esta "sociología de la militancia" busca estudiar las transformaciones de la vida política a nivel de los itinerarios, carreras o trayectorias de militantes (nivel de análisis micro-sociológico); la historia de las organizaciones (nivel de análisis meso-sociológico), y la evolución del compromiso y la participación política en las sociedades contemporáneas (nivel de análisis macro-sociológico), poniendo el foco de atención tanto en la perspectiva de los agentes como en las condiciones desde las cuales estos han dado curso a su compromiso militante, revelando procesos sociosimbólicos y socioestructurales de base, vinculados al cambio o la reproducción social. El enfoque acerca de las mutaciones de las formas del compromiso político y de la militancia resulta atractivo como herramienta heurística sociológica, pues abre nuevas pistas de investigación a un fenómeno -el del activismo, la militancia-, que ha sido abordado en la historia reciente de Chile prácticamente solo desde el punto de vista de la recolección de testimonios en el marco de la historiografía de las violaciones a los derechos humanos o de la evaluación de las políticas públicas de reparación y "reconciliación” en esta materia. Enfoques, por

El concepto de "carrera militante" está inspirado en el interaccionismo simbólico de Goffman y resulta útil para dar cuenta del carácter procesual y dinámico del compromiso militante, como una sucesión de etapas que participan de la construcción de las identidades sociales y que suponen un análisis del compromiso en el tiempo. Utilizando este concepto para la investigación de la acción colectiva, Olivier Fillieule lo define como la actividad social que articula tiempos de reclutamiento, de mantenimiento del compromiso y de defección. Al respecto, ver: Fillieule, "Devenirs militants". 
lo general, muy ligados a la represión vivida, lo que ha tendido a recortar e invisibilizar un proceso social, de dimensiones socioestructurales y sociosimbólicas, a escalas micro y mesosociales, rico en permanencias y cambios, que tiene un antes, un durante y un después de la dictadura militar, y que ha afectado de modos diversos las trayectorias de los agentes sociales, el funcionamiento de las antiguas y nuevas organizaciones en las que participan, así como del conjunto del campo de los derechos humanos.

A este respecto, la perspectiva sociológica desarrollada por Pierre Bourdieu, a veces llamada "estructuralismo genético" (Cosas dichas 26), ofrece un instrumental teórico relevante, en tanto se trata de una aproximación al estudio de lo social que problematiza las antinomias clásicas de separación e incluso antagonismo entre el modo de conocimiento objetivista y subjetivista, lo simbólico y lo material, lo teórico y lo práctico. En su lugar, Bourdieu propone una economía unificada de las prácticas, uniendo el enfoque fenomenológico con el estructural, al interior de un modo de investigación integrado y epistemológicamente coherente. Intentando trascender la reducción de la sociología a quedar atrapada en alguno de los polos de la física objetivista de las estructuras materiales o de la fenomenología constructivista, el "estructuralismo genético" -O "praxeología social" de acuerdo a Loïc Wacquant (Bourdieu y Wacquant)-, postula una ontología no cartesiana, en la que objeto y sujeto, intención y causa, así como materialidad y representación simbólica no quedan separados. Con ello, Bourdieu se aleja de las concepciones que consideran a los individuos como soportes pasivos que se adaptan mecánicamente a su entorno, así como de la visión de que la sociedad sería el producto de decisiones contingentes tomadas por actores competentes, en un mundo social inmediatamente dado y significado.

La aproximación de Bourdieu resulta útil para abordar los objetivos de la investigación del campo de derechos humanos que supere el "modelo familiar", por su perspectiva relacional y disposicional, que toma en consideración el análisis de las posiciones sociales, las disposiciones (o los habitus) y las tomas de posición (las elecciones que los agentes sociales llevan a cabo en los ámbitos de la práctica). Desde esta perspectiva, la investigación permite dar con las potencialidades inscritas en el cuerpo de los agentes y en la estructura de las situaciones en las que estos actúan, tratando de aprehender unas estructuras y mecanismos como los principios de construcción -principios de formación o generación- del espacio social 
(o los mecanismos de reproducción de este espacio), señalando "las diferencias reales que separan tanto las estructuras como las disposiciones (los habitus), cuyo principio no se indaga en las singularidades de las naturalezas -o de las 'almas'-, sino en las particularidades de historias colectivas diferentes” (Bourdieu, Razones prácticas 13).

El habitus sería ese principio generador y unificador que retraduce las características instrínsecas y relacionales de una posición en un estilo de vida unitario, es decir, un conjunto unitario de elección de personas, de bienes y de prácticas. Los habitus se diferencian y son diferenciantes, son principios generadores de prácticas distintas y distintivas, pero "también son esquemas clasificatorios, principios de clasificación, principios de visión y de división, aficiones diferentes. Establecen diferencias (...) pero no son las mismas diferencias para unos y otros". Así, "existir en un espacio, ser un punto, un individuo en un espacio, significa diferir, ser diferente" (Bourdieu, Razones prácticas 21).

Al concebir al mundo social como espacio, Bourdieu pone la atención en las estructuras de diferencias que cabe comprender cuando se elabora el principio generador que fundamenta estas diferencias en la objetividad. Este principio es la estructura de la distribución de las formas de poder o de las especies de capital eficientes en el universo social considerado -y que, por lo tanto, varían según los lugares y los momentos-. La estructura o el principio generativo no es, en consecuencia, inmutable, por lo que se puede realizar un análisis dinámico de la conservación y de la transformación de la estructura de distribución de las propiedades actuantes, y con ello del espacio social (Bourdieu, Raz̧ones prácticas 49).

El espacio social así considerado es un campo, es decir, a la vez un campo de fuerzas, cuya necesidad se impone a los agentes que se han adentrado en él, y un campo de luchas dentro del cual los agentes se enfrentan, con medios y fines diferenciados según su posición en la estructura del campo de fuerzas, contribuyendo de este modo a conservarla o a transformarla.

Podemos, entonces, investigar el espacio de posiciones y la construcción de estructuras objetivas, así como la experiencia de los propios agentes sociales que pueden explicar tales estructuras. La lógica del campo involucra las posibilidades que tienen los individuos en un momento dado; el valor social adjudicado a los atributos que poseen, como el lugar de formación, por ejemplo, en función de las categorías de percepción y de apreciación socialmente construidas. A su vez, se involucra el "clima de época" en tanto 
una serie de conocimientos prácticos acerca de las propias posibilidades e imposibilidades de los individuos.

Para elaborar el espacio social es necesario:

[T]omar en cuenta las diferentes especies de capital cuya distribución determina la estructura del espacio social (...). El espacio social se organiza según tres dimensiones fundamentales: en la primera dimensión, los agentes se distribuyen según el volumen global de capital que poseen, mezclando todas las especies de capital; en la segunda, según la estructura de este capital, es decir según el peso relativo del capital (...); en la tercera, según la evolución en el tiempo del volumen y de la estructura de su capital (Bourdieu, Ražones prácticas 29).

Esta mirada nos resulta útil para poder realizar la "sociología de la militancia" de quienes adhieren y participan de los movimientos, agrupaciones y asociaciones al interior de un campo específico, los derechos humanos, permitiéndonos establecer las posiciones diferenciales de quienes lo integran (queremos precisamente dar con la heterogeneidad del movimiento) de acuerdo a su trayectoria social, especialmente del universo de agentes sociales que constituyen sus grupos dirigentes en el tiempo, y observar en la eventual variación de su perfil social y político las modificaciones en la configuración del campo ${ }^{4}$.

A este respecto, la propuesta de Bourdieu también nos sirve para concentrarnos en los grupos dirigentes, en lo que podríamos llamar una "sociología de las élites" 5 , toda vez que los conceptos de campo, espacio

4 La noción de configuración es heredera de la aproximación de Norbert Elias, quien la define como "entramados de interdependencia constituidos por individuos" y "modelos cambiantes compuestos por jugadores entendidos como un todo, por todo su yo, por el conjunto de intercambios y sus mutuas relaciones" (45).

A este respecto, la definición más corriente de élite que se utiliza en sociología es la de Wright Mills, quien la entiende como "minoría del poder de los círculos políticos, económicos y militares que por lo menos tienen consecuencias nacionales. En la medida en que se deciden los acontecimientos nacionales, la elite del poder está constituida por quienes lo deciden" (25). Consideramos que en el caso de esta investigación no aplica exactamente este concepto, por lo que hemos optado de hablar de "grupos dirigentes" para especificar que se trata del universo de agentes sociales que ocupan una posición diferencial en el espacio social de los movimientos, agrupaciones y asociaciones de 
social y capital nos permiten investigar las eventuales modificaciones en los principios de jerarquización y de legitimación, así como las lógicas de acción que estructuran y dan sentido al mundo social en el que ocurren determinadas prácticas, y el papel que al interior de determinado espacio social juegan los distintos tipos de capital que pueden movilizar los agentes.

Bourdieu, entonces, representa

el mundo social bajo la forma de un espacio, de muchas dimensiones, construido bajo la base de principios de diferenciación o de distribución constituidas por el conjunto de las propiedades activas dentro del universo social considerado, es decir, capaces de conferir a su detentador la fuerza, el poder en ese universo. Los agentes y los grupos de agentes son definidos de este modo por sus posiciones relativas en ese espacio. (...) En la medida en que las propiedades seleccionadas para construir este espacio son propiedades activas, se le puede describir también como un campo de fuerzas; es decir, como un conjunto de relaciones de fuerza objetivas que se imponen a todos aquellos que entran al campo y que son irreductibles a las intenciones de los agentes individuales o incluso a las interacciones directas entre los agentes (Bourdieu, "El espacio social").

Las propiedades activas que, en el respectivo campo, han sido seleccionadas como principios de construcción del espacio social son las diferentes especies de poder o capital, que representan un poder sobre el campo (en un momento dado) y sobre el producto acumulado del trabajo anterior. Las especies de capital, de este modo, son poderes que definen las probabilidades de beneficio en un campo dado.

En efecto, junto a la comprensión de la estructura del campo, la lógica de su funcionamiento y de sus transformaciones, se puede describir la relación que se establece entre los agentes singulares, por lo tanto sus

defensa de los derechos humanos respecto de los demás miembros que les permite, por la lógica del campo y de los principios de jerarquía (dados los tipos y cantidad de capitales de que disponen y de los usos que le dan), ocupar una posición de poder al interior del entramado de relaciones sociales de las que participan. Por otra parte, por las connotaciones diversas que reviste el significante "élite", nos parece que el concepto de "grupos dirigentes" logra una expresión más neutra para el tipo de investigación que nos ocupa. 
habitus, y las fuerzas del campo, y que se objetiva en una trayectoria. Aquí la trayectoria, a diferencia de una biografía corriente, "describe la serie de posiciones sucesivamente ocupadas [por el agente social] en los estados sucesivos del campo (...), dando por supuesto que sólo en la estructura de un campo, es decir una vez más relacionalmente, se define el sentido de estas posiciones sucesivas" (Bourdieu, Ražones prácticas 72).

Para efectos de ilustrar este punto, se puede citar una investigación que realizaron Pierre Bourdieu y Monique de Saint Martin sobre el episcopado francés, en la que proponen un modelo de análisis no sustancialista, destinado a captar la dinámica interna de ese grupo de representación, que busca construir una imagen de unidad y de homogeneidad, y la investigación está en condiciones de resaltar sus heterogeneidades. A partir del análisis del "background" de los prelados, distinguen dos perfiles, lo que les permite mostrar que no hay la mentada homogeneidad, sino la coexistencia de representantes de varias generaciones y cambios de la composición social en el tiempo. Bourdieu enfatiza que no existe una relación mecánica entre el origen social y la trayectoria seguida, sin perjuicio de que el primero constituye el principio que determina propiedades como el habitus de clase, que a su vez determina la posición ocupada y ésta las tomas de posición (Ferrari 529).

De acuerdo a la lógica del campo, la transformación de los cargos en el tiempo hace que las disposiciones de los agentes que los ocupan sean diferentes en distintos momentos. Dado que la sociedad se transforma, se demandan nuevas capacidades para acceder a los cargos. En el ejemplo de la investigación de Bourdieu ya citada, una vez que se ubican los perfiles y las condiciones de posibilidad para que los prelados alcancen ciertas posiciones, el análisis del episcopado se realiza en relación con el campo religioso, donde se da la presencia de otros integrantes (profesionales, laicos, teólogos, políticos) que compiten con los obispos. De este modo, el modelo propone partir de la distribución de atributos, colocarlos en relación con las condiciones de posibilidad y la lógica del campo, y observar las tensiones internas del propio campo, las competencias y la manera de competir en que se ponen en juego los recursos de los agentes.

Aplicado al caso chileno, es posible levantar la hipótesis de que, en el marco del proceso de institucionalización de la causa de los derechos humanos en el espacio nacional, se ha ido transformando el espacio social de su práctica, en una creciente profesionalización que, entre otros 
aspectos, se expresa en que los principios de diferenciación al interior del campo de los derechos humanos han ido variando, en una tendencia en la que el tipo y cantidad de capital que tuvo una posición predominante en épocas pasadas -el capital simbólico o de prestigio- estaría cediendo ante la valorización del capital cultural y social ${ }^{6}$. Si esto fuera así, y es lo que las investigaciones empíricas desde esta perspectiva estarían en condiciones de demostrar, se debiera observar una modificación de las "carreras militantes" al interior del campo de los derechos humanos, con la consiguiente modificación de quienes ingresan a los grupos dirigentes, dado que los principios de jerarquización y legitimidad han cambiado. Este cambio en la configuración del campo no solo indicaría una profesionalización creciente sino, al mismo tiempo, evidenciaría que a la temática de los derechos humanos no solo tienen acceso -como tarea exclusiva- los familiares de víctimas y luchadores sociales caídos por acción del terrorismo de Estado, sino que una ciudadanía más amplia se hace cargo de ella en modo creciente, abriéndose un campo sociopolítico en disputa, lleno de invención y herencia, memoria y futuro.

Bibliografía

Agrupación Chilena de Familiares de Detenidos Desaparecidos. Un Camino de Imágenes que Revelan y se Rebelan Contra una Historia No Contada: 20 Años de Historia de la Agrupación de Familiares de Detenidos Desaparecidos de Chile. Santiago: Corporación Agrupación de Familiares de Detenidos Desaparecidos, 1997. Citado como AFDD. Impreso.

Bennani-Chraïbi, Mounia y Olivier Fillieule (eds.). Resistencia y protesta en las sociedades musulmanas.Barcelona: Bellaterra, 2004. Impreso.

Brito, Alexandra Barahona de. Human Rights and Democratization in Latin America: Uruguay and Chile. Nueva York: Oxford University Press, 1997. Impreso.

6 En algunas investigaciones en que se aplica este concepto para el caso de organizaciones o movimientos le llaman "capital militante", en cuanto a todo lo que remite al saber militante, a la experiencia militante, a la socialización militante y al sentimiento de competencia militante. Al respecto, Matonti y Popeau (4-11). 
Bourdieu, Pierre y Loïc Wacquant. Una invitación a la sociología reflexiva.

Buenos Aires: Siglo XXI, 2005. Impreso.

Bourdieu, Pierre. Cosas dichas. Barcelona: Gedisa, 1996. Impreso. "El espacio social y la génesis de las 'clases". Sociología y cultura. México: Grijalbo/CONACULTA, 1990. Impreso.

Razones prácticas. Sobre la teoría de la acción. Barcelona: Anagrama, 1997. Impreso.

Brett, Sebastian. Chile Unsettled Business: Human Rights in Chile at the Start of the Frei Presidency. Nueva York: Human Rights Watch Americas, 1994. Impreso.

Cuadros, Daniela: "Engagement et expertise des droits de l'homme. Trente ans de mobilisations au nom des victimes de la dictature chilienne". Mobilisations de victims, Sandrine Lefranc y Lilian Mathieu (dirs.). Rennes: Presses Universitaires de Rennes, 2009. Impreso.

Davies, James Chowning. "Toward a Theory of Revolution". American Sociological Review 27.1 (febrero 1962): 5-19. Impreso.

Elias, Norbert. El proceso de civilización (Investigaciones sociogenéticas y psicogenéticas). Madrid/ México: Fondo de Cultura Económica, 1987. Impreso.

Ferrari, Marcela. "Prosopografía e historia política. Algunas aproximaciones". Antiteses, vol. 3, n 5 (jan-jun. de 2010): 529-550. Impreso.

Fillieule, Olivier. "Propositions pour une analyse processuelle de l'engagement individuel. Post scriptum". Revue franacaise de science politique 51.1-2 (febrero-abril 2001). Impreso.

"Devenirs militants". Sciences Humaines 144 (diciembre 2003).

FrüHling, Hugo, ed. Derechos humanos y democracia. La contribución de las organizaciones no gubernamentales. Santiago: Instituto Interamericano de Derechos Humanos, 1991. Impreso.

Fuentes, Marta. "Les femmes contre la dictadure". Inprécor 225, 14 de diciembre 1987. Impreso.

Goffman, ERving. Internados. Ensayos sobre la situación social de los enfermos mentales. Buenos Aires: Amorrurtu Editores, 2004. Impreso. 
Groppo, Bruno y Patricia Flier (comp.). La imposibilidad del olvido. Recorridos de la memoria en Argentina, Chile y Uruguay. La Plata: Editorial Al Margen, 2001.

Gurr, Ted. Why Men Rebel?. Princeton, NJ: Princeton University Press, 1970. Impreso.

HawkINS, DARREn G. International human rights and authoritarian rule in Chile. Lincoln/Londeres: University of Nebraska Press, 2002. Impreso.

Hipsher, Patricia. "Democratic Transitions as Protest Cycles: Social Movement Dynamics in Democratizing Latin America". The Social Movement Society. David Meyer y Sidney Tarrow (eds.). Maryland: Rowman \& Litllefield Publishers, 1998. Impreso.

Kitschelt, Herbert. "Political Opportunity and Political Protest: AntiNuclear Movements in Four Countries". British Journal of Political Science 65 (1986). Impreso.

Kriesi, Hanspeter. "New Social Movements and Political Opportunities in Western Europe". European Journal of Political Research 22 (1992). Impreso.

Le Bon, Gustave. Psychologie des foules. París: Presses Universitaires de France, 1947 [1895]. Impreso.

Lira, Elizabeth \& Brian Loveman. "Derechos Humanos en la Transición 'Modelo': Chile 1988-1999”. En Paul Drake e Iván Jaksic, (eds.). El Modelo Chileno, Democracia y Desarrollo en los Noventa. Santiago, Chile: LOM Ediciones, 1999. 339-374. Impreso.

Loveman, Mara. "Collective Action in Contexts of Extreme Risk: Southern Cone Human Rights Movements in Comparative Perspective" (paper), 1997.

"Southern Cone Human Rights Movements in Comparative Perspective", ponencia presentada en el congreso de la Latin American Studies Association, Chicago, 1998. Inédito.

McAdam, Doug. The Political Process and the Development of Black Insurgency, 1930-1970. Chicago: University of Chicago Press, 1982. Impreso.

McAdam, Doug, John McCarthy y Mayer Zald. Movimientos sociales: perspectivas comparadas. Madrid: Istmo, 1999. Impreso.

McAdam, Doug, Sydney Tarrow y Charles Tilly. Dynamics of contention. Nueva York: Cambridge University Press, 2001. Impreso. 
Mills, Charles Wright. La élite del poder. México: Fondo de Cultura Económica, 1987. Impreso.

MARIOt, Nicolás. “La efervescencia social como problema de investigación”. Revista Política 44 (Otoño 2005). Impreso.

Matonti, Frédérique y Franck Popeau. "Le capital militant. Essai de définition". Actes de la recherche en sciences sociales 155 (2004/5): 4-11.

O’Donnell, Guillermo y Philippe Schmitter (eds.). Transitions from authoritarian rule: Tentative Conclusions. Baltimore: John Hopkins University Press, 1986. Impreso.

Oliveira, Wilson José Ferreira. "As Condições Sociais de Institucionalização de Perspectivas Teóricas no Estudo das Mobilizações Ambientalistas". Ponencia presentada en el III Encuentro de ANPPAS, Brasilia, 23 al 26 de mayo de 2006. Inédito.

Olson, Mancur. La lógica de la acción colectiva, bienes públicos y la teoría de grupos. México: Limusa/Noriega, 1992. Impreso.

Orellana, Patricio. "Los organismos de derechos humanos en Chile hacia 1985”. El movimiento de Derechos Humanos en Chile, 1973-1990. Patricio Orellana y Elizabeth Q. Hutchison. Santiago: Centro de Estudios Políticos Latinoamericanos Simón Bolivar, 1991. 7-68. Impreso.

Oxhorn, Philip. Organizing Civil Society: The Popular Sectors and the Struggle for Democracy in Chile. University Park: Pennsylvania State University Press, 1991. Impreso.

Palestro, Sandra. "Mujeres en movimiento 1973-1989". Documento de trabajo Facultad Latinoamericana de Ciencias Sociales 14 (1991). Impreso.

Schneider, Cathy Lisa. Shantytown Protest in Pinochet's Chile. Philadelphia: Temple University Press, 1995. Impreso.

Smelser, Neil. Teoría del comportamiento colectivo. México: Fondo de Cultura Económica, 1989 [1963].

Snow, D. A. y Benford, R. D. "Master frames and cycles of protests". Morris, Aldon D. y McClerg Müller, Carol (eds.). Frontiers in social movement theory. New Heaven, Yale University Press, 1992.

SNOW, D. A. ET AL. "Frame alignment process, micromobilization, and movement participation". American Sociologial Review (1986).

TARde, Gabriel. L'opinion et la foule. París: Félix Alcan, 1901. Impreso. 
Tarrow, Sidney. Poder en movimiento. Movimientos sociales, acción colectiva y politica de masas en el estado moderno. Madrid: Alianza, 1977. Impreso.

Touraine, Alain. Movimientos sociales de hoy. Actores y analistas. Barcelona: Hacer, 1990. Impreso.

Valenzuela, María. La mujer en el Chile militar. Santiago: Ediciones Chile y América/CESOC/ACHIP, 1987. Impreso.

Vecchiol, Virginia. "Derechos Humanos y Compromiso Militante. Un recorrido por la constitución de esta causa a través del activismo de profesionales del derecho". Etnografías Contemporáneas 3 (2007). Impreso.

"Os Trábalhos pela Memoria. Um esboco do campo dos Direitos Humanos na Argentina através da construcao social da categoría vítima do terrorismo de Estado". Tesis para optar al grado de Magíster en la UFRJ/Museu Nacional/PPGAS. Río de Janeiro, 2000. Impreso.

Vidal, Hernán. El Movimiento Contra la Tortura "Sebastián Acevedo": Derechos Humanos y la Producción de Símbolos Nacionales Bajo el Fascismo Chileno. 2002. Santiago: Mosquito Editores. Impreso.

Dar la vida por la vida. Agrupación Chilena de Familiares de Detenidos Desaparecidos. Santiago: Mosquito Editores, 1996. Impreso.

Recepción: 03.01.2014

Aceptación: 13.01.2014 